\title{
An Operational Safety Evaluation Method for Manned Transport Aircraft and Large UAV in Mixed Airspace
}

\author{
Xin Ma, ${ }^{1}$ Xiaoqiang Zhang $\mathbb{D}^{2}{ }^{2}$ Huawei Wang, ${ }^{1}$ Songbin Ding, ${ }^{1}$ and Xia $\mathrm{Li}^{2}$ \\ ${ }^{1}$ Nanjing University of Aeronautics and Astronautics, Nanjing 211106, China \\ ${ }^{2}$ Civil Aviation Flight University of China, Guanghan 618307, Sichuan, China \\ Correspondence should be addressed to Xiaoqiang Zhang; xqzhanguestc@163.com
}

Received 24 December 2020; Revised 13 February 2021; Accepted 12 March 2021; Published 9 April 2021

Academic Editor: Bosheng Song

Copyright (C) 2021 Xin Ma et al. This is an open access article distributed under the Creative Commons Attribution License, which permits unrestricted use, distribution, and reproduction in any medium, provided the original work is properly cited.

To improve the predictive ability in trajectory of large unmanned aerial vehicle (UAV) and the calculation performance in complicated circumstances with mixed airspace, multiple aircraft types, and joint operations, the concept of phased trajectory deviation (PTD) is introduced and a corresponding minimal interval algorithm (PTD-MI) is set up. This algorithm is capable of deriving the minimal interval between various aircraft types according to the crosswind impacts and the UAV characteristics at different flight phases and thus achieves the effective safety evaluation in airspace operation. To demonstrate the rationality and generality of the proposed algorithm, several simulation experiments are conducted. Based on the experimental results, flight procedure protection area is plotted by PTD-MI algorithm and compared with that generated by Ground-Based Augmentation System (GBAS). Results indicate that the proposed algorithm is capable of deriving a more scientific basis for airspace assignment and outperform GBAS in dealing with wide-area space problems. And, compared with GBAS, PTD-MI algorithm shows a more stable calculation performance and is easier to output the results. PTD-MI algorithm is proposed under the flight safety regulation designed by the International Civil Aviation Organization (ICAO) and designed to provide effective technical supports for the safe and normal operations of aircrafts.

\section{Introduction}

At present, many older manned aircraft, because of the poor performances of the aircraft, the small commercial payload space of the aircraft, and the deficiency of aviation telecommunication equipment [1], have gradually been modified into large unmanned aircraft, which are used in low-altitude cargo transportation and general aviation flights $[2,3]$.

In the research field of UAV track prediction and operation safety assessment, the current research accumulation is as follows. First of all is the study of risk factors. By sorting out the unsafe incidents, we can conclude the causes of the accidents. Wang and Chen provided a hybrid optimization algorithm with directivity [4], Zhao et al. proposed a case study for diagnosis [5], Zhang used the learning model to supplement experiments and demonstrate the imperfections in the algorithm and case diagnosis $[6,7]$. In this way, the graded management method of accident factors has been established. Second is the study of risk degree. According to the hierarchical management of accident factors, factor screening and weight design have become the focus of safety evaluation model [8]. In the preliminary studies, the evaluation schemes were mostly built around the SHELL model, which basically realized the accident risk assessment [9]. At this stage of the study, Chen proposed a Hawks optimization scheme based on the computer system $[10,11]$, Li et al. provide a global optimization strategy [12], and Xia et al. provided intelligent systems [13], and the results of their research explain and justify the implementation of the optimization scheme. Shen used a grasshopper optimizer to enhance the adaptability of previous studies $[14,15]$ so that the various levels of risk assessment parameters obtained a specific design basis. Third is the study of flight path prediction. According to the available data, the method of graded management of accident factors and the scheme of risk assessment have been initially established [16]. Chen 
studied early accident diagnosis and provided research ideas for parameter selection and evaluation [17], $\mathrm{Hu}$ studied the defects of accident diagnosis and proposed the use of multiplicity selection method to carry out simulation experiments [18], and Zhang et al. established a method to show the application effect of experimental results by using Image Dehazing (ID) technology $[19,20]$. The next step of research will mainly focus on the collection of original data of accident factors [21]. According to different research objects and evaluation criteria, flight path prediction research is mainly used for flight path planning [22]. Fourth is the study on the accuracy of evaluation results. Since all industries have accident evaluation programs, but the requirements for accuracy of evaluation results and quantitative methods are different, civil aviation flight safety assessment emphasizes precision and control [23]. In a study conducted in recent years, Zhao proposed the application of image segmentation and processed the evaluation results graphically [24], Zhang et al. proposed the concept of integer programming to make the evaluation results more accurate in the graphic display [25], and Zhao et al. proposed Improved Ant Colony Optimization (Improved ACO) to improve the display degree and scope of evaluation results $[26,27]$. These research results make the simulation results and evaluation results more applicable to the real civil flight practice field. At present, a breakthrough has been made in the study of flight safety evaluation; however, in dealing with problems such as high precision, large scope, and complex airspace structure, the existing algorithms have low computational performance [28], or cannot carry out evaluation for specific environment, and lack a calculation method with high adaptability [29]. At the same time, the existing research lacks the countermeasures to the joint operation. Even so, in terms of algorithm and comparative experiment design, the research results are rich. In the future, experiments and argumentation can be carried out under the premise of optimal design, and evaluation basis and rules can be summarized.

In the current practical operation and production process, more and larger UAV and manned aircraft are carrying out joint operation missions [30]. In this mixed airspace, flight safety assessment is a difficult work, and the main difficulties lie in the choice of parameter types and the use of evaluation criteria [31]. At the same time, transform old manned aircraft into large UAV; it has been gradually realized at this stage, and this kind of transformation is more and more [32], and the number of old aircraft is also large [33]. As a result, general aviation missions at low altitudes will increasingly performed using large UAV; if a safety assessment method can be developed which is suitable for the joint operation of large UAV and manned aircraft in mixed airspace $[34,35]$, these findings can improve the flight safety of general aviation and promote the strategy of further opening up the low-altitude airspace [36].

This paper takes operational safety assessment as the research direction and tries to establish a trajectory prediction model. This model can be used to evaluate the operational safety trend in the low-altitude airspace, and the evaluation scope should cover the local airspace of the airport, and the evaluation results should meet the ICAO requirements for the accuracy of risk assessment.

\section{Problem Description}

First of all, because of the preflight planning stage, it is impossible to accurately obtain all kinds of conditions during the actual flight, such as airspace situation, meteorological information, and flight environment [37].Those differences in the preplanning and implementation leads to the result between the planned trajectory and the actual trajectory which is different [38]. According to the conflict probability value stipulated by ICAO [39], the navigation equipment accuracy of large UAV is used to analyze various influencing factors under the operating environment [40], and the fusion algorithm is established for the overall airspace to calculate and obtain the interval safety value that should be maintained between aircrafts, so as to complete the evaluation of flight conflict [41]. As described above, the current model lacks the analysis and processing of aircraft performance parameters and does not consider the turning radius trajectory deviation or the linear [42]. If the flight procedure design specification of transport aircraft is used for analysis, many unfavorable factors will be brought so that the calculated results cannot be used for correct evaluation [43]. Therefore, the design of PTD-MI algorithm needs to avoid the above adverse factors.

Secondly, the airport has a wide range of local airspace. If ground equipment is used for trajectory observation and prediction [44], the increase of observation distance will lead to the increase of evaluation error [45]. Therefore, the design of PTD-MI algorithm needs to consider the optimization of evaluation error.

Finally, the evaluation results derived from the algorithm can easily show the application effect.

\section{Improved Computational Model}

According to ICAO regulations, calculate flight envelope on the mission to carry out the plan, and all running implementation stages need to meet standards, including the conflict between aircraft probability which are restricted to $1.5 \times 10^{-8}$ (accident/flight hours) [46]; the data can be seen as the flight safety guarantee value, and it can be used to represent the current conflict between the probability of aircraft operating. In the design of the improved model, the large UAV is used as the experimental object [47].

3.1. Established Model of Turning Stage. As experimental objects, large UAVs are generally equipped with Global Positioning System, which can autonomously and accurately search for preset airway points, and have the ability to fly along the preset path [48]. All of them have no flight technical tolerance and do not need to set the time margin for the pilot response. In the actual flight process, compared with the transport aircraft, the experimental subjects need to focus on the data such as lift-off point position, turning speed, turning slope, and flight mission altitude, which are the main parameters for the formation of flight trajectory of 
large UAV. Assuming rated cruising speed $v_{1}$ and turning slope $\gamma_{1}$, there is a relationship between rated turning radius $R_{1}$ and flight parameters under current flight conditions:

$$
R_{1}=\frac{v_{1}^{2}}{g \tan \gamma_{1}} \text {. }
$$

Assuming that the real slope is $\gamma_{2}$ and the cruising speed $v_{2}$ is arbitrary, the normal distribution relationship is established:

$$
\begin{aligned}
& F_{\gamma}\left(\gamma_{2}\right)=\frac{1}{\sqrt{2 \pi \sigma_{\gamma}}} \exp \left(-\frac{\left(\gamma_{1}-\gamma_{2}\right)^{2}}{2 \sigma_{\gamma}^{2}}\right), \\
& F_{v}\left(v_{2}\right)=\frac{1}{\sqrt{2 \pi \sigma_{v}}} \exp \left(-\frac{\left(v_{1}-v_{2}\right)^{2}}{2 \sigma_{v}^{2}}\right) .
\end{aligned}
$$

Assuming that the center of the circle is $O$, the model expression of the turning trajectory is established:

$$
\left\{\begin{array}{l}
x_{2}=x_{1}+R_{0} \cos \alpha, \\
y_{2}=y_{1}-R_{0} \sin \alpha .
\end{array}\right.
$$

Assuming that the center of the allowed maximum turning trajectory is represented by $O_{1}\left(x_{1}, y_{1}\right)$ and the center of the real turning trajectory is represented by $\mathrm{O}_{2}\left(x_{2}, y_{2}\right)$, it is assumed that the trajectory of the real flight overlaps with the preset trajectory, which is generated by the true course angle, represented by $\alpha$. Because global positioning system is widely used for navigation and positioning in the field of civil aviation transportation, its civil precision is usually about 50 meters [49]. At the same time, equipment error, installation error, and aerodynamic error exist in real environment and experiment. After taking all kinds of errors into consideration, a normal distribution relationship is established. Assuming that the maximum permissible heading angle is $\alpha_{1}$ and the true heading angle is $\alpha_{2}$, then

$$
F_{\alpha}\left(\alpha_{2}\right)=\frac{1}{\sqrt{2 \pi \sigma_{\alpha}}} \exp \left(-\frac{\left(\alpha_{1}-\alpha_{2}\right)^{2}}{2 \sigma_{\alpha}^{2}}\right) \text {. }
$$

According to the relevant provisions of aircraft operation attached to ICAO Annex 6, the $\sigma_{\alpha}$ value is $2.58^{\circ}$, without considering the case of $\alpha_{1}=\alpha_{2}$, and the expression of $\alpha$ is established as follows:

$$
\alpha=\left\{\begin{array}{l}
\pi-\arctan \left(\frac{y_{1}}{x_{1}}\right), \\
2 \pi-\arctan \left(\frac{y_{1}}{x_{1}}\right) .
\end{array}\right.
$$

3.2. Established Model of Crosswind Influence. Assuming that the experimental object is affected by the crosswind with wind speed of WS, according to the conservative principle, omnidirectional wind is used to represent the crosswind; then,

$$
R_{2}=R_{1}+\frac{W S}{\mu},
$$

where, under the omnidirectional wind, the true turning radius is $R_{2}$ and the angle to complete the turn is $\mu$. Therefore, under the influence of the above turning slope, height, and other factors, the trajectory deviation component $S_{r d}$ can be expressed as follows:

$$
S_{r d}=x_{L}-x_{2}+R_{1}
$$

where $x_{L}$ is a known quantity. The value of $x_{L}$ is the value of the actual flight position of the experimental object in the coordinate system $\mathrm{O}_{2}$.

3.3. Established Model of Straight Flight. Generally, in the straight flight stage, due to the influence of navigation equipment precision and wind, there are differences between the actual flight path and the preflight planned path. In the total flight time of the experiment, the experimental subjects spent more flight time in the straight way and less flight time in the turning way. If, in the advance path planning stage, the deviation caused by straight flight and turn flight is calculated separately and integrated, then this approach will increase the workload of preflight preparation [50]. According to the conservative principle of ICAO in the way of safety assessment, the deviation value of the straight line segment and the turning segment can be calculated uniformly, to obtain the total amount of deviation satisfying the two modes of flight, thus ensuring the adaptability of the calculation results. 
Assumptions exist in space plane position point $A_{3}\left(x_{3}(t, t \Delta), y_{3}(t, t \Delta)\right)$, it means, in the preset trajectory points, to allow maximum deviation, there is a position point $A_{4}\left(x_{4}(t, t \Delta), y_{4}(t, t \Delta)\right) \cos ^{-1} \theta$, and it means the current real trajectory point position. Because the experiment task is to fly straight and then temporarily not consider height variation, $d$ means the distance between the two points above, flight time for $t \Delta$; then, we can get the space position of expression:

$$
\left\{\begin{array} { l } 
{ x _ { 3 } ( t , t _ { \Delta } ) = \varepsilon _ { 3 x } + ( - d _ { 3 } + \int _ { 0 } ^ { t + t _ { \Delta } } v _ { 3 } ( t ) \mathrm { d } t ) \cdot \operatorname { c o s } \phi , } \\
{ y _ { 3 } ( t , t _ { \Delta } ) = \varepsilon _ { 3 y } , }
\end{array} \left\{\begin{array}{l}
x_{4}\left(t, t_{\Delta}\right)=\varepsilon_{4 x} \cdot \cos \phi-\varepsilon_{4 y} \cdot \sin \phi+\cos \beta \cos \phi\left(-d_{4}+\int_{t_{\Delta}}^{t+t_{\Delta}} v_{4}(t) \mathrm{d} t\right) \\
y_{4}\left(t, t_{\Delta}\right)=\varepsilon_{4 x} \cdot \sin \phi+\varepsilon_{4 y} \cdot \cos \phi+\cos \beta \sin \phi\left(-d_{4}+\int_{t_{\Delta}}^{t+t_{\Delta}} v_{4}(t) \mathrm{d} t\right)
\end{array}\right.\right.
$$

where $i$ is the number of the experimental subject, $\beta$ is the position point with the maximum allowable deviation, $\phi$ is the real position point, there is an included angle between points $\beta$ and $\phi$ and any point $O$ on the preplanned trajectory in the flight direction, and $\varepsilon$ is the coordinate system position tolerance. In the current case, the trigonometric relationship consisting of a line of points $O$, flight direction, and trajectory deviation values is established.

Suppose the time interval is $t \Delta$; then, $\left|x_{34}(t, t \Delta)\right|$ and $\left|y_{34}(t, t \Delta)\right|$, respectively, represent the position relationship of $\beta$ and $\phi$ with respect to the position point $O$, and the relationship is as follows:

$$
\left\{\begin{array} { l } 
{ x _ { 3 4 } ( t , t _ { \Delta } ) = \varepsilon _ { 3 x } - ( \varepsilon _ { 4 x } \operatorname { c o s } \phi - \varepsilon _ { 4 y } \operatorname { s i n } \phi ) + S _ { 3 4 x } ( t , t _ { \Delta } ) , } \\
{ y _ { 3 4 } ( t , t _ { \Delta } ) = \varepsilon _ { 3 y } - ( \varepsilon _ { 4 y } \operatorname { s i n } \phi + \varepsilon _ { 4 y } \operatorname { c o s } \phi ) + S _ { 3 4 y } ( t , t _ { \Delta } ) , }
\end{array} \left\{\begin{array}{l}
S_{34 x}\left(t, t_{\Delta}\right)=\cos \phi\left(-d_{3}+\int_{0}^{t+t_{\Delta}} v_{3}(t) \mathrm{d} t\right)-\cos \phi \cos \beta\left(-d_{4}+\int_{t_{\Delta}}^{t+t_{\Delta}} v_{2}(t) \mathrm{d} t\right) \\
S_{34 y}\left(t, t_{\Delta}\right)=-\cos \beta \sin \phi\left(-d_{4}+\int_{t_{\Delta}}^{t+t_{\Delta}} v_{4}(t) \mathrm{d} t\right)
\end{array}\right.\right.
$$

where position point $O$ is the initial point, the experimental subject and point $O$ exist at points $S_{34 x}(t, t \Delta)$ and $S_{34 y}(t, t \Delta)$, and points $S_{34 x}(t, t \Delta)$ and $S_{34 y}(t, t \Delta)$ are symmetric with the preplanned trajectory, the three establish trigonometric relations. Then, when $t \Delta$ is a specific value that can be measured and obtained, the distance between two points can be obtained:

$$
S_{34}\left(t, t_{\Delta}\right)=\sqrt{S_{34 x}^{2}\left(t, t_{\Delta}\right)+S_{34 y}^{2}\left(t, t_{\Delta}\right)} .
$$

According to the above expression, the distance between the real flight trajectory position point and point $O$ should be greater than the minimum safety distance, so the distance between the two points should be wider than 0 , which is a positive number. If that is true, that means the mission can continue, in which the subject is at an appropriate range, and it satisfies the probability of safety collision and is not easy to have collision and conflict. If the conclusion is equal to 0 or less than 0 , it means that the experimental subject is in the restricted range, or already in the dangerous area. According to the conservative principle, the expression of the minimum critical deviation component in the straight flight stage is obtained, and the relationship is as follows:

$$
\left|S_{34}\left(t, t_{\Delta}\right)\right|=S_{L \min } \text {. }
$$

After derivation, the total deviation $M$ in the turning flight stage and the straight flight stage is obtained, and the expression is as follows:

$$
S_{r d}+S_{L \min }=M
$$

3.4. Established Model of Safe Operation. The initial design of the model is as follows:

$$
\left\{\begin{array}{l}
w_{1}=w_{m x}+w_{n x} \\
w_{2}=w_{m y}+w_{n y} \\
w_{3}=w_{m z}+w_{n z}
\end{array}\right.
$$

where $w_{m x}, w_{m y}, w_{m z}$ and $w_{n z}, w_{n y}, w_{n z}$ are the dimensions of transport aircraft and experimental objects and the parameters are length, wingspan, and height in order. If the transport aircraft and the experimental subject regard each other as points, when their respective initial models are in contact, it can be considered that the operation conflict has been generated and the safety is affected (see Figure 1).

The improved model is established by comprehensively considering all the safe operation parameters, and the relationship is as follows: 


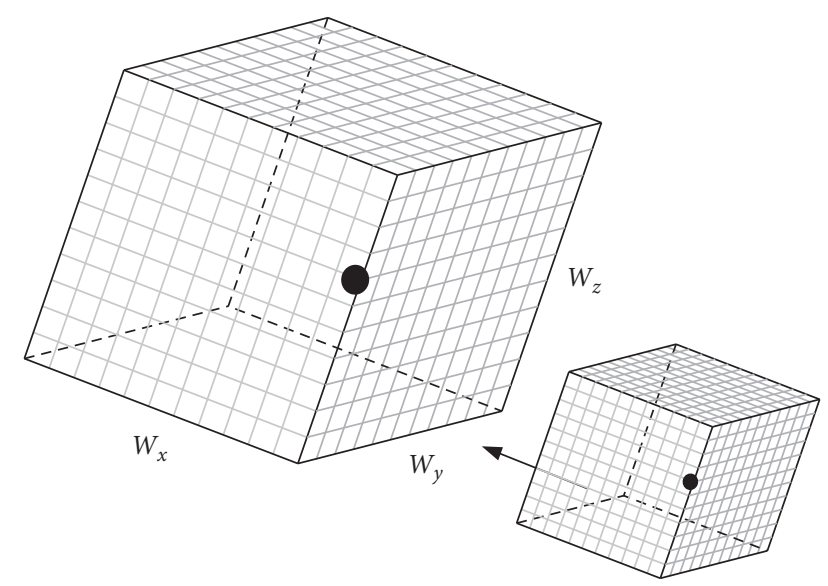

Figure 1: The initial model and model contact.

$$
\begin{aligned}
G= & \frac{A}{M}\left(w_{1}+\frac{U_{s} w_{2}}{V_{s}}\right) P\left(1+\frac{W_{s} w_{2}}{V_{s} w_{3}}\right) \eta \\
& +\frac{B}{M}\left(w_{3}+\frac{U_{o} w_{2}}{V_{o}}\right) P\left(1+\frac{W_{o} w_{2}}{V_{o} w_{3}}\right) \eta,
\end{aligned}
$$

where $G$ is the value of conflict probability obtained through calculation by importing the security operation parameters into the improved model, $\eta$ is the operating sorties of transport aircraft per hour, $A$ represents for the number of experimental subjects (UAV), which is consistent with the flight direction and flight trend of transport aircraft in the current airspace, and $B$ also represents for the number of experimental subjects (UAV), which is remains opposite with the transport aircraft in flight direction and trend within the current airspace. Assuming that when the conflict occurs if the flight trend of the experimental subject and the transport aircraft is consistent, then $U_{s}, V_{s}$, and $W_{s}$ are used to represent the velocity data parallel to the flight direction, perpendicular to the flight direction, and above the flight direction. Similarly, $U_{o}, V_{o}$, and $W_{o}$ are data under the trend of remaining relative flight. $P$ is the probability that the transport aircraft and the experimental subject (UAV) fly over each other.

In order to make the experimental conclusion adaptable to civil aviation manned transport aircraft, the parameter composition and constant setting in the model should meet the requirements of ICAO on flight standards and safe operation, etc [51]. Computational and research objects mainly include experimental objects (UAV), other aircraft similar in type to experimental objects, and civil aviation manned transport aircraft. Assume that $\varphi_{1}$ is the quality of flight mission execution, $\varphi_{2}$ is the pilot operation technology, and $\varphi_{3}$ is the probability of operating failure per 100,000 hours, and statistics of real operation data in recent ten years is shown in Table $1[52,53]$. Because the research objects are civil aviation manned transport aircraft and experimental objects (UAV), civil aviation transport flight has a strict set of retention barriers, as required by the law and regulations, and it is permissible for a civilian transport aircraft with a fault to fly missions; according to the conservative principle, the operation failure probability of transport aircraft can refer to the ICAO regulation, namely, the maximum number of failures per 100,000 hours allowed.

According to the preset data and real data in the table, the expression of $\varphi_{1}$ is obtained as follows:

$$
\varphi_{1}=\sum_{i=1}^{n} E_{i} T_{i},
$$

where $E_{i}$ is the applicability of flight status and $T_{i}$ is the number of aircraft in the experimental airspace. In addition, according to formula (15), $E_{i}$ is used to represent the failure data per 100,000 hours and $T_{i}$ is used to represent the failure interval data. So, the expression of $\varphi_{2}$ can be obtained.

Pilot's flying skills are mainly reflected in the civil aviation manned transport aircraft, and UAV is not affected by this factor and can directly use the former accident ratio $9.62 \%$; then, $\varphi_{3}=1-0.0962=0.9$.

To sum up, according to the provisions of ICAO, one conflict in safe operation will be recorded as two accidents, and the evaluation model of safe operation conflict will be established. The expression $R E$ is as follows:

$$
R E=2\left(1-\varphi_{1}\right) \cdot \varphi_{2} \cdot\left(1-\varphi_{3}\right) \cdot G .
$$

If the calculated result of $R E$ is set as the probability value of safe operation conflict, which is constant and meets the requirements of ICAO for safe operation, the improved model is used for analysis and calculation. Assuming that the relative motion states of the transport aircraft and the experimental subject are unchanged, the structure of the airspace remains unchanged, the operating conditions remain unchanged, then $(A+B)$ can be regarded as the sum of the number of aircraft in the current airspace so that the airspace density data can be obtained. This calculation result is a quantified airspace management conclusion and meets the flight standard of safe operation conflict probability.

\section{Design of Simulation Experiment}

An airport in the south of China was selected for the simulation experiment, and the flight performance data of the research object were referred to Airbus 320 and AT200, as shown in Table $2[54,55]$. Because the AT200 and the same class of UAV, which are large fixed-wing UAV, must take off from civilian airports, so, in flight, take-off climb and approach phase is more prone to conflict. The parameters were added into the improved calculation model, the influence of flight trajectory deviation is considered, and the conflict probability value of safe operation is considered, so the minimum interval value between the research objects can be calculated to obtain the turning flight stage and the straight flight stage. The above calculation results can be used as the capacity conclusion of the flight airspace, which is a quantitative result.

Considering the airport information, consider the route and route point information and then start the design of the simulation experiment. Normally, July and August are the busiest months for transport aircraft; select the hourly flight flow in the low-altitude airspace within the research airspace, 
TABLE 1: The maintenance actions and costs corresponding to different maintenance levels.

\begin{tabular}{lcccc}
\hline Type & $\begin{array}{c}\text { Number of serious failures per } \\
\text { 100,000 flight hours }\end{array}$ & $\begin{array}{c}\text { MTBF (hour) } \\
\text { Airbus 320 (no }\end{array}$ & $\begin{array}{c}\text { Applicability of flight } \\
\text { status (\%) }\end{array}$ & Task completion schedule (\%) \\
statistical NEO) & 0.20 & 48.5 & 12 & 85 \\
Boeing 737NG (800) & 0.20 & 49.2 & 12 & 85 \\
AT-200 & 12 & 29.5 & 96 & 86 \\
Y-5 (UAV) & 9 & 23.4 & 87 & 78 \\
Predator & 34 & 44.1 & 73 & 79 \\
Pioneer & 248 & 19.2 & 77 & 83 \\
Hunter & 15 & 10.9 & 96 & 81 \\
\hline
\end{tabular}

TABle 2: Parameters of simulation experiment.

\begin{tabular}{lccc}
\hline Type & Data & Type & Data \\
\hline$V(\mathrm{~km} / \mathrm{h})$ & 650 & $w_{m x}(\mathrm{~m})$ & 37.57 \\
$\Gamma$ & 45 & $w_{m y}(\mathrm{~m})$ & 34.10 \\
$\sigma_{v}$ & 15 & $w_{m z}(\mathrm{~m})$ & 11.76 \\
$\sigma_{\alpha}$ & 2 & $w_{n z}(\mathrm{~m})$ & 11.84 \\
$P$ & 0.5 & $w_{n y}(\mathrm{~m})$ & 12.80 \\
$\mathrm{H}$ & - & $w_{n z}(\mathrm{~m})$ & 4.04 \\
$V_{S}$ or $V_{O}$ & 5.23 & $U_{s}$ & 3 \\
$W_{S}$ or $W_{O}$ & 0.58 & $U_{o}$ & 497 \\
$W_{S}(\mathrm{~km} / \mathrm{h})$ & 30 & Altitude $(\mathrm{m})$ & 3200 \\
\hline
\end{tabular}

using this data as the value of $\eta$. According to the influence of the cutting angle and heading angle on the experimental design, three different angle relationships are established.

Design 1. When the two planes are flying in the same direction and the transport aircraft is in the departure and climbing stage, in this case, transport aircraft would travel from low-altitude airspace to medium altitude airspace, or higher altitude, so set the maximum $\beta=15^{\circ}$ and $\varphi=20^{\circ}$, and this is according to ICAO operating standard.

Design 2. When the two planes are flying face to face and the transport aircraft is in the arrived stage, in this case, the transport aircraft has a high speed, and set $\beta=5^{\circ}$ and $\varphi=20^{\circ}$.

Design 3. When the two planes are flying face to face and both planes are flying horizontally, in this case, the flight status of transport aircraft is relatively stable, and the possibility of sudden changes in operating conditions is low, and set $\beta=0^{\circ}$ and $\varphi=15^{\circ}$.

The above three conditions were established to simulate the situation where the transport aircraft and the UAV had a high probability of conflict, increase the redundancy of experimental results, and adhere to the conservative principle.

According to the design of the experiment, parameters are added into the model, and the conclusion can be obtained through equations (12) and (16):

$$
\begin{aligned}
& \text { (i) The first case, } \quad M_{(1)}>3.116 \mathrm{~km} \text {, } \\
& (A+B)_{1}=1.203 \times 10^{-3}\left(\text { frame } / \mathrm{km}^{3}\right)
\end{aligned}
$$

(ii) The second case, $M_{(2)}>4.214 \mathrm{~km}$, $(A+B)_{2}=0.895 \times 10^{-3}\left(\right.$ frame $\left./ \mathrm{km}^{3}\right)$

(iii) The third case, $M_{(3)}>3.762 \mathrm{~km}$, $(A+B)_{3}=1.054 \times 10^{-3}\left(\right.$ frame $\left./ \mathrm{km}^{3}\right)$

The experimental results can be made into a graph to show the relationship between the conflict probability of safe operation and the interval between the aircraft, and the relation of density is obtained, as shown in Figures 2 and 3.

\section{Description of Application and Innovation}

According to three groups of experiments, when the experimental subjects are in the stage of arrival and departure flight, they will have a large space change and speed change for climbing. For this reason, the amount that can be used to adjust the heading angle is reduced, resulting in insufficient space for the flight path to be used for adjustment or avoidance in preflight preparation, and there will a high probability of collision, and it is very likely to happen to intrude into another airspace. At the same time, it also shows that, in this operating state, the density of joint operating aircraft needs to keep low, which conforms to the objective facts. It can be concluded that, in the low-altitude airspace, the adjustment variation of heading angle can provide data support for calculating the probability of collision.

Similar evaluation models mostly exist in the collaborative evaluation between ground operating equipment and aircraft, and the main reason is that both civil aviation and general aviation aircraft perform missions in low-altitude airspace using conventional or visual flight procedures, depending on the location of ground equipment, ground visual references, and route point setting points and lack of accurate positioning ability and monitoring equipment. As a result, the data of the evaluation model contains the positioning tolerance value, so the practical guiding significance is not sufficient, and does not apply to the evaluation of the mixed airspace for large UAV and manned aircraft [56].

In order to reflect the advantages of PTD-MI algorithm in dealing with wide-area space problems, according to the design of simulation experiment, the data in Table 2 were added into the evaluation model of GBAS, and the calculation results are shown in Table 3.

Among them, the experimental results under the GBAS model are divided into two situations: the results within 15 kilometers and the results outside 15 kilometers. Because 


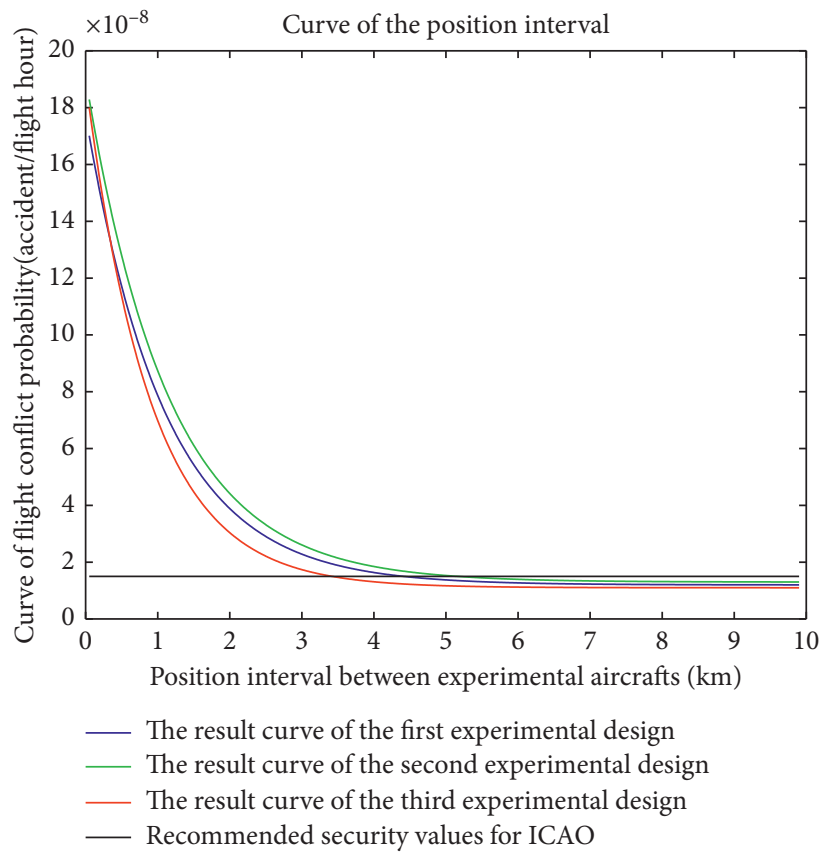

Figure 2: Curve of the position interval.

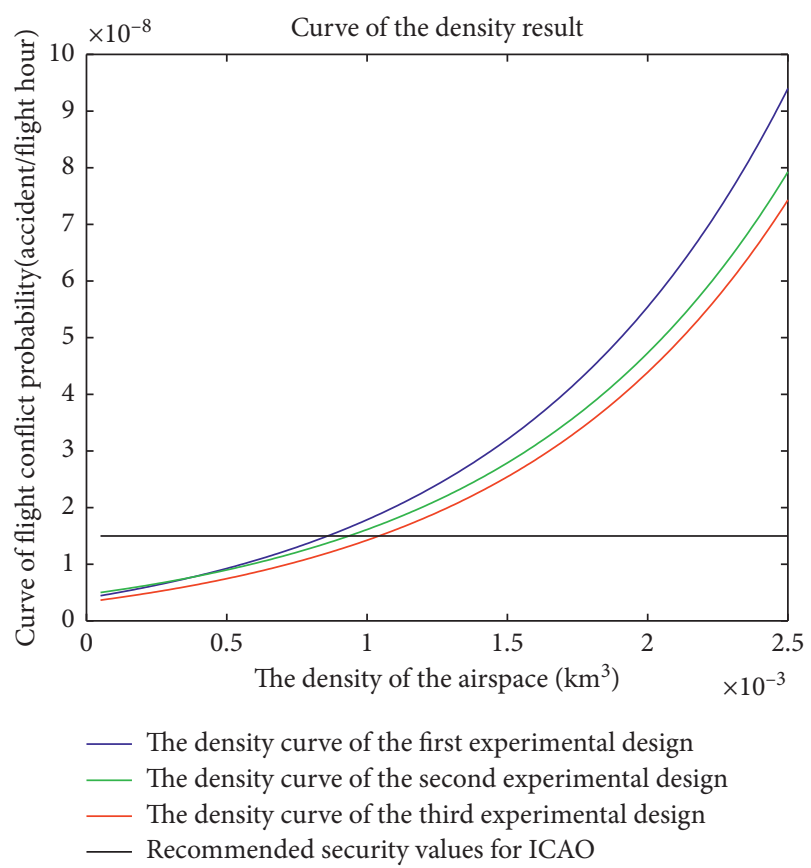

Figure 3: Curve of the density result.

the distance between ground equipment and aircraft increases, the positioning error of ground equipment also increases. According to the results of the third case of experiments, the flight procedure protection area is drawn, as shown in Figures 4 and 5.

As shown in the picture, there are three colors: white, gray, and black, and they represent the operating areas based on the above two evaluation conclusions. The operation area is divided into three layers: the first layer is the area where flight missions can be carried out, the second layer is the buffer area where the possibility of conflict needs to be carefully handled, and the third layer is the area where UAV is prohibited to fly. Taking the approach stage and low-altitude airspace as examples, the altitude of low-altitude airspace shall be calculated as below 6000 meters (inclusive), and the spatial planning quantity of simulation experiment results is shown in Table 4. In Figures 6 and 7, the parameters of simulation experiment are set as follows: the elevation of the airport is 1150 meters, and the average mountain height within the range of the approach stage is 1372.26 meters.

The application effect of the experimental results shows that due to the use of the PTD-MI model, the volume of the first layer airspace increases by $10.04 \%, 9.54 \%$, and $8.09 \%$, which indicates that the flight area of the large UAV is expanded, and the application effect is excellent.

The reasons for the above results can be summarized as three points. First, the GBAS evaluation model evaluates the working data of ground equipment [57]. Thie equipment uses $30 \mathrm{MHz}-300 \mathrm{MHz}$ electromagnetic wave for data transmission to realize navigation and positioning functions [58]. Therefore, there will be an attenuation of efficiency when information is transmitted in a large area, which leads to the fact that the $M$ value is not a constant quantity. Its positioning tolerance value will increase with the expansion of the evaluation airspace [59], so it is not suitable for the research of position interval in the overall airspace. This method is suitable for evaluation in a small space such as near the runway. Second, the ground equipment cannot be built at the midpoint of the runway entrance, so the deviation of heading angle will inevitably occur [60], and when the flight value is smaller, the accuracy of the data is higher, so the error is very obvious due to the existence of deviation. Thirdly, relevant parameters 
TABLE 3: Comparison of experimental results based on the two algorithms.

\begin{tabular}{|c|c|c|c|}
\hline $\begin{array}{l}\text { Experimental design sequence } \\
\text { number }\end{array}$ & Types of experimental results & $\begin{array}{l}\text { Model of GBAS (within } 15 \text { kilometers; outside } \\
15 \text { kilometers) }\end{array}$ & PTD-MI \\
\hline \multirow[b]{2}{*}{ The first case } & The total deviation $M, \mathrm{~km}$ & $>2.588 ;>3.844$ & $>3.116$ \\
\hline & $\begin{array}{l}\text { Total number of aircraft }(A+B), \\
\text { frame } / \mathrm{km}^{3}\end{array}$ & $1.281 \times 10^{-3} ; 0.982 \times 10^{-3}$ & $1.203 \times 10^{-3}$ \\
\hline \multirow[b]{2}{*}{ The second case } & The total deviation $M, \mathrm{~km}$ & $>3.602 ;>5.013$ & $>4.214$ \\
\hline & $\begin{array}{l}\text { Total number of aircraft }(A+B) \text {, } \\
\text { frame } / \mathrm{km}^{3}\end{array}$ & $0.962 \times 10^{-3} ; 0.615 \times 10^{-3}$ & $0.895 \times 10^{-3}$ \\
\hline \multirow[b]{2}{*}{ The third case } & The total deviation $M, \mathrm{~km}$ & $>3.116 ;>4.607$ & $>3.762$ \\
\hline & $\begin{array}{l}\text { Total number of aircraft }(A+B), \\
\text { frame } / \mathrm{km}^{3}\end{array}$ & $1.138 \times 10^{-3} ; 0.807 \times 10^{-3}$ & $1.054 \times 10^{-3}$ \\
\hline
\end{tabular}

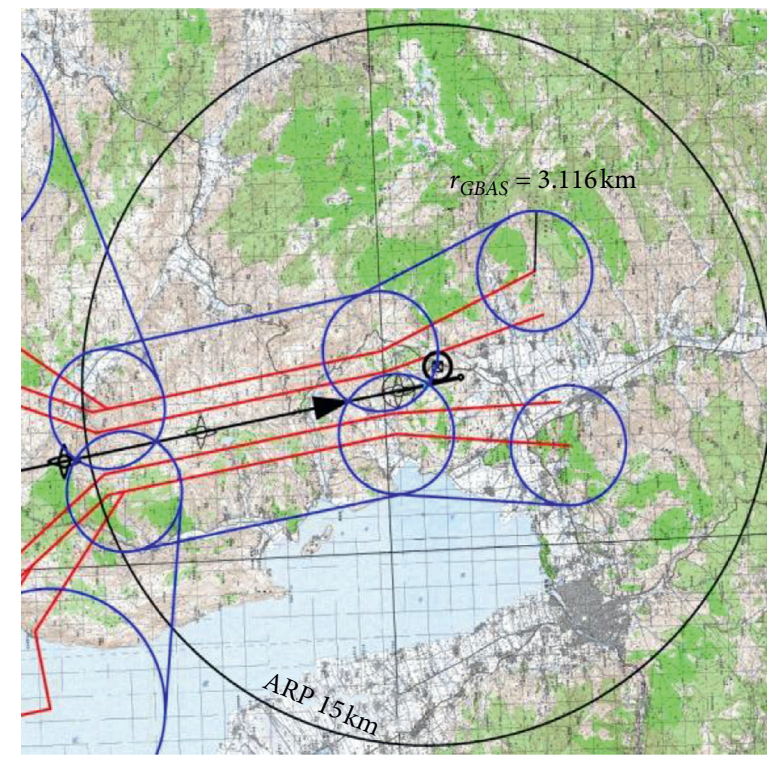

FIgURE 4: The result of approach phase based on the GBAS model.

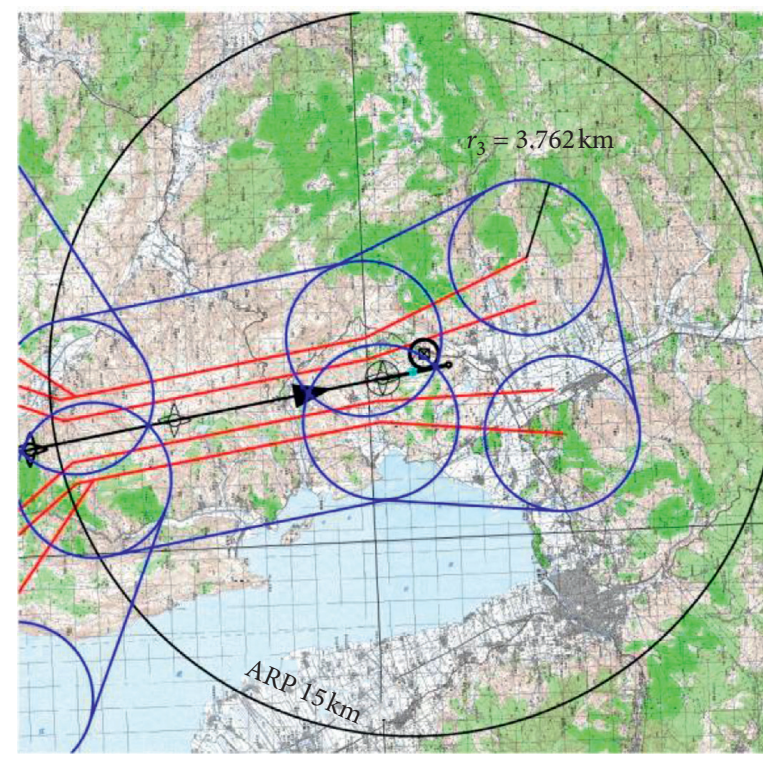

FIgure 5: The result of approach phase based on PTD-MI. 
TABLE 4: Spatial planning quantity based on simulation experiment results.

\begin{tabular}{lccccc}
\hline Layer number & \multicolumn{2}{c}{$\begin{array}{c}\text { Simulation experiment of } \\
\text { the first case }\left(\mathrm{km}^{3}\right)\end{array}$} & \multicolumn{2}{c}{$\begin{array}{c}\text { Simulation experiment of } \\
\text { the second case }\left(\mathrm{km}^{3}\right)\end{array}$} & \multicolumn{2}{c}{$\begin{array}{c}\text { Simulation experiment of } \\
\left.\text { the third case }(\mathrm{km})^{3}\right)\end{array}$} \\
& GBAS & PTD-MI & GBAS & PTD-MI & GBAS \\
The first layer & 8464.73 & 9409.55 & 8016.88 & 8863.63 & 8542.81 \\
The second layer & 1092.78 & 910.38 & 1302.83 & 1059.76 & 1169.32 \\
The third layer & 2387.95 & 2387.95 & 2387.95 & 2387.95 & 2387.95 \\
\hline
\end{tabular}

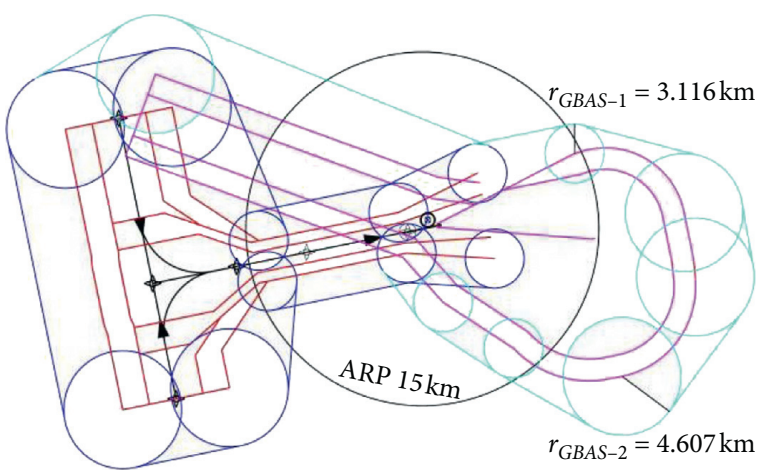

FIGURE 6: The results of mixed airspace based on the GBAS model.

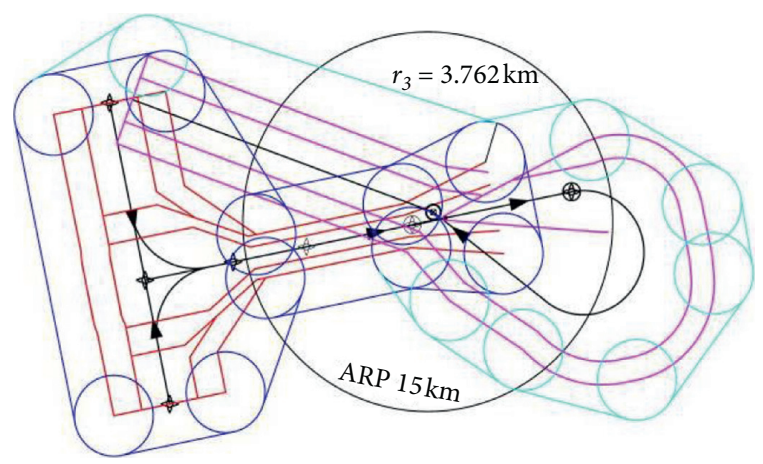

FIgURE 7: The result of mixed airspace based on PTD-MI.

based on satellite positioning are not included in the ground equipment evaluation model, which will greatly increase the inaccuracy of the calculation conclusions in the application of space problems. Based on the above three reasons, it can be found that the experimental design takes data accuracy, safety margin and mixed airspace operation into full consideration [61]. The conclusion obtained by the PTD-MI algorithm calculation makes the $M$ value in a more reasonable range and improves the scientific nature of the density value.

There are two innovations about the PTD-MI algorithm. First, the model fully considers the flight characteristics of the turning stage and the straight stage and adds the flight attitude data into the evaluation model to obtain the results. This is the first time to study such a problem. Second, take the flight conflict safety value recommended by ICAO as the basis of experimental design [62], the first discussion of flight planning in the mixed airspace, including conflict assessment between large UAV and manned aircraft, and these conclusions are applicable to preflight planning of flight missions in the overall airspace.

\section{Summary and Conclusions}

In this paper, the concept of PTD is introduced, and a corresponding PTD-MI algorithm is set up. According to the actual operation and production situation, the simulation experiment is designed and the experimental results are shown, and the experimental results reflect the application effect of operation safety evaluation. In addition, the experimental results of the GBAS model are compared and analyzed, and the conclusions are as follows:

(1) The PTD-MI algorithm is consistent with the requirements of rationality and generality in the actual operation, which is conducive to maintaining the accuracy of the experimental results. The algorithm maintains the characteristics of large UAV at all flight stages and under the influence of crosswind, and it provides an optimal basis to deal with airspace planning problems and widens the application range of PTD-MI algorithm.

(2) In the simulation experiments, the PTD-MI algorithm shows excellent and effective computing performance. In the prediction of the flight path within the local airspace of the airport, the acceptable calculation results can be provided. By comparing and analyzing the results of PTD-MI algorithm and GBAS algorithm, the superiority of PTD-MI algorithm in dealing wide-area space problems is shown.

(3) Due to the reference of ICAO flight standard parameters, the calculation results derived from PTDMI algorithm are applicable to the operation safety evaluation of civil aviation.

For future works, we recommend applications of the proposed method to other UAV systems [63-67], adaptive control techniques [68-72], applications of optimization methods such as differential evolution, particle swarm, whale optimizer, differential search, and other optimizers for optimizing parts of the process [73-78], distributed optimization [79], and other optimization forms such as robust, memetic, many objective, multiobjective, and fuzzy optimization [80-87].

\section{Data Availability}

The data used to support the findings of the study are available within the article. 


\section{Conflicts of Interest}

The authors declare no conflicts of interest.

\section{Authors' Contributions}

X. M. and X.Z. conceptualized the study; X. M. developed methodology; X. Z. helped with software; H. W. and S. D. validated the study, X. M. carried out formal analysis; X. L. investigated the study; X. M. collected resources; X. M. carried out data curation; X. M. wrote the original draft; X. Z. reviewed and edited the article; X. M. visualized the study; H. W. and S. D. supervised the study; X. M. administrated the project; X. M. helped with funding acquisition. All authors have read and agreed to the published version of the manuscript.

\section{Acknowledgments}

This research was supported by Civil Aviation Administration of China, development foundation educational talents program (no.14002600100018J034) and general foundation of Civil Aviation Flight University of China (J2018067). The funders had no role in the design of the study, in the collection, analyses, or interpretation of data, in the writing of the manuscript, or in the decision to publish the results.

\section{References}

[1] X. Zhang, J. J. Zhang, and S. F. Wu, "Aircraft monitoring by the fusion of satellite and ground ADS-B data," Acta Astronautica, vol. 11, pp. 398-405, 2017.

[2] H. Li, A. P. Teixeira, and C. Guedes Soares, "A two-stage failure mode and effect analysis of offshore wind turbines," Renewable Energy, vol. 162, pp. 1438-1461, 2020.

[3] J. J. Sofonia, S. Phinn, C. Roelfsema, F. Kendoul, and Y. Rist, "Modelling the effects of fundamental UAV flight parameters on LiDAR point clouds to facilitate objectives-based planning," ISPRS Journal of Photogrammetry and Remote Sensing, vol. 149, pp. 105-118, 2019.

[4] M. Wang and H. Chen, "Chaotic multi-swarm whale optimizer boosted support vector machine for medical diagnosis," Applied Soft Computing, vol. 88, Article ID 105946, 2020.

[5] X. Zhao, X. Zhang, Z. Cai et al., "Chaos enhanced grey wolf optimization wrapped ELM for diagnosis of paraquat-poisoned patients," Computational Biology and Chemistry, vol. 78, pp. 481-490, 2019.

[6] Y. Zhang, "Towards augmented kernel extreme learning models for bankruptcy prediction: algorithmic behavior and comprehensive analysis," Neurocomputing, vol. 430, pp. 185-212, 2020.

[7] X. Zhang, "Robust feature learning for adversarial defense via hierarchical feature alignment," Information Sciences, vol. 10, p. $42,2020$.

[8] N. A. Golilarz, H. Gao, R. Kumar, L. Ali, Y. Fu, and C. Li, "Adaptive wavelet based MRI brain image de-noising," Frontiers in Neuroscience, vol. 14, p. 728, 2020.

[9] N. A. Golilarz, H. Gao, W. Ali, and M. Shahid, "Hyperspectral remote sensing image de-noising with three dimensional wavelet transform utilizing smooth nonlinear soft thresholding function," in Proceedings of the 15th
International Computer Conference on Wavelet Active Media Technology and Information Processing, pp. 142-146, Chengdu, China, December 2018.

[10] H. Chen, A. A. Heidari, H. Chen, M. Wang, Z. Pan, and A. H. Gandomi, "Multi-population differential evolutionassisted Harris hawks optimization: framework and case studies," Future Generation Computer Systems, vol. 111, pp. 175-198, 2020.

[11] Y. Xu, H. Chen, J. Luo, Q. Zhang, S. Jiao, and X. Zhang, "Enhanced Moth-flame optimizer with mutation strategy for global optimization," Information Sciences, vol. 492, pp. 181203, 2019.

[12] C. Li, L. Hou, B. Y. Sharma et al., "Developing a new intelligent system for the diagnosis of tuberculous pleural effusion," Computer Methods and Programs in Biomedicine, vol. 153, pp. 211-225, 2018.

[13] J. Xia, H. Chen, Q. Li et al., "Ultrasound-based differentiation of malignant and benign thyroid Nodules: an extreme learning machine approach," Computer Methods and Programs in Biomedicine, vol. 147, pp. 37-49, 2017.

[14] L. Shen, H. Chen, Z. Yu et al., "Evolving support vector machines using fruit fly optimization for medical data classification," Knowledge-Based Systems, vol. 96, pp. 61-75, 2016.

[15] C. Yu, "SGOA: annealing-behaved grasshopper optimizer for global tasks," Engineering with Computers, vol. 1, pp. 1-28, 2021.

[16] M. A. Lotufo, L. Colangelo, and C. Perez-Montenegro, "UAV quadrotor attitude control: an ADRC-EMC combined approach," Control Engineering Practice, vol. 11, pp. 13-22, 2018.

[17] H.-L. Chen, G. Wang, C. Ma, Z.-N. Cai, W.-B. Liu, and S.-J. Wang, "An efficient hybrid kernel extreme learning machine approach for early diagnosis of Parkinson's disease," Neurocomputing, vol. 184, pp. 131-144, 2016.

[18] J. Hu, "Orthogonal learning covariance matrix for defects of grey wolf optimizer: insights, balance, diversity, and feature selection," Knowledge-Based Systems, vol. 213, Article ID 106684, 2020.

[19] X. Zhang, T. Wang, W. Luo, and P. Huang, "Multi-level fusion and attention-guided CNN for image dehazing," IEEE Transactions on Circuits and Systems for Video Technology, vol. 99, p. 1, 2020.

[20] X. Zhang, "Pyramid channel-based feature attention network for image dehazing," Computer Vision and Image Understanding, vol. 197-198, Article ID 103003, 2020.

[21] H. Li, H.-Z. Huang, Y.-F. Li, J. Zhou, and J. Mi, "Physics of failure-based reliability prediction of turbine blades using multi-source information fusion," Applied Soft Computing, vol. 72, pp. 624-635, 2018.

[22] H. Li, H. Diaz, and C. G. Soares, "A developed failure mode and effect analysis for floating offshore wind turbine support structures," Renewable Energy, vol. 164, pp. 133-145, 2020.

[23] J.. Marion, P. Letortu, and T.. Claire, "UAV survey of a coastal cliff face-Selection of the best imaging angle," Measurement, vol. 24, pp. 110-117, 2019.

[24] D. Zhao, "Chaotic random spare ant colony optimization for multi-threshold image segmentation of 2D Kapur entropy," Knowledge-Based Systems, vol. 216, Article ID 106510, 2020.

[25] X. Zhang, M. Fan, D. Wang, P. Zhou, and D. Tao, “Top-k feature selection framework using robust 0-1 integer programming," IEEE Transactions on Neural Networks and Learning Systems, vol. 31, p. 1, 2020.

[26] X. Zhao, D. Li, B. Yang, C. Ma, Y. Zhu, and H. Chen, "Feature selection based on improved ant colony optimization for 
online detection of foreign fiber in cotton," Applied Soft Computing, vol. 24, pp. 585-596, 2014.

[27] X. Zhang, D. Wang, Z. Zhou, and Y. Ma, "Robust low-rank tensor recovery with rectification and alignment," IEEE Transactions on Pattern Analysis and Machine Intelligence, vol. 34, p. 1, 2019.

[28] R. U. Khan, X. Zhang, R. Kumar, A. Sharif, N. A. Golilarz, and M. Alazab, "An adaptive multi-layer botnet detection technique using machine learning classifiers," Applied Sciences, vol. 9, no. 11, p. 2375, 2019.

[29] N. K. Jain, U. Nangia, and J. Jain, "A review of particle swarm optimization," Journal of The Institution of Engineers (India): Series B, vol. 99, no. 4, pp. 407-411, 2018.

[30] S. Aggarwal and N. Kumar, "Path planning techniques for unmanned aerial vehicles: a review, solutions, and challenges," Computer Communications, vol. 149, pp. 270-299, 2020.

[31] W. Peng, Z. S. Ye, and N. Chen, "Bayesian deep-learningbased health prognostics toward prognostics uncertainty," IEEE Tra. onInd. Ele.vol. 67, no. 3, pp. 2283-2293, 2019.

[32] Z. Bai, L. Y. Ling, and T. Han, "Research on attiude stabilitu of small UAV," Computer Simulation, vol. 466, pp. 16-20, 2017.

[33] N. A. Golilarz, H. Gao, and H. Demirel, "Satellite image de-noising with harris hawks meta heuristic optimization algorithm and improved adaptive generalized Gaussian distribution threshold function," IEEE Access, vol. 7, pp. 57459-57468, 2019.

[34] N. A. Golilarz, M. Mirmozaffari, T. A. Gashteroodkhani et al., "Optimized wavelet-based satellite image de-noising with multi-population differential evolution-assisted harris hawks optimization algorithm," IEEE Access, vol. 8, pp. 133076$133085,2020$.

[35] G. Xiang and C. Guedes Soares, "Improved dynamical modelling of freely falling underwater cylinder based on CFD," Ocean Engineering, vol. 211, p. 107538, 2020.

[36] H. Li and C. G. Soares, "Reliability analysis of floating offshore wind turbines support structure using hierarchical Bayesian network," in Proceedings of the 29th European Safety and Reliability Conference, pp. 2489-2495, Hannover, Germany, September 2019.

[37] M. Riahi Manesh and N. Kaabouch, "Analysis of vulnerabilities, attacks, countermeasures and overall risk of the automatic dependent surveillance-broadcast (ADS-B) system," International Journal of Critical Infrastructure Protection, vol. 19, pp. 16-31, 2017.

[38] M. Wang, H. Chen, B. Yang et al., "Toward an optimal kernel extreme learning machine using a chaotic moth-flame optimization strategy with applications in medical diagnoses," Neurocomputing, vol. 267, pp. 69-84, 2017.

[39] L. Hu, G. Hong, J. Ma, X. Wang, and H. Chen, “An efficient machine learning approach for diagnosis of paraquat-poisoned patients," Computers in Biology and Medicine, vol. 59, pp. 116-124, 2015.

[40] X. Xu and H.-1. Chen, "Adaptive computational chemotaxis based on field in bacterial foraging optimization," Soft Computing, vol. 18, no. 4, pp. 797-807, 2014.

[41] Y. Zhang, "Boosted binary Harris hawks optimizer and feature selection," Engineering with Computers, vol. 25, p. 26, 2020.

[42] J. Tu, H. Chen, J. Liu et al., "Evolutionary biogeography-based whale optimization methods with communication structure: towards measuring the balance," Knowledge-Based Systems, vol. 212, p. 106642, 2021.
[43] N. A. Golilarz, N. Robert, J. Addeh, and A. Salehpour, "Translation invariant wavelet based noise reduction using a new smooth non-linear improved thresholding function," Computational Research Progress in Applied Science and Engineering, vol. 3, no. 3, pp. 104-108, 2017.

[44] M. Yazdi, F. Nikfar, and M. Nasrabadi, "Failure probability analysis by employing fuzzy fault tree analysis," International Journal of Intelligent Engineering and Systems, vol. 8, no. 2, pp. 1177-1193, 2017.

[45] M. Khatami and S. H. Zegordi, "Coordinative production and maintenance scheduling problem with flexible maintenance time intervals," Journal of Intelligent Manufacturing, vol. 28, no. 4, pp. 857-867, 2017.

[46] C. Liu, N. X. Luo, and L. B. Lu, "Rotorcraft route planning based on artificial potential field," Construction Simulator, vol. 81, pp. 101-105, 2017.

[47] C.-G. Huang, H.-Z. Huang, and Y.-F. Li, "A bidirectional LSTM prognostics method under multiple operational conditions," IEEE Transactions on Industrial Electronics, vol. 66, no. 11, pp. 8792-8802, 2019.

[48] Y. Liu, Y. Chen, and T. Jiang, "On sequence planning for selective maintenance of multi-state systems under stochastic maintenance durations," European Journal of Operational Research, vol. 268, no. 1, pp. 113-127, 2018.

[49] C. D. Dao and M. J. Zuo, "Optimal selective maintenance for multi-state systems in variable loading conditions," Reliability Engineering \& System Safety, vol. 166, pp. 171-180, 2017.

[50] W. Shan, "Double adaptive weights for stabilization of moth flame optimizer: balance analysis, engineering cases, and medical diagnosis," Knowledge-Based Systems, vol. 13, Article ID 106728, 2020.

[51] H. Yu, "Dynamic Gaussian bare-bones fruit fly optimizers with abandonment mechanism: method and analysis," Engineering with Computers, pp. 1-29, 2020.

[52] X. Zhang, R. Jiang, T. Wang, and J. Wang, "Recursive neural network for video deblurring," IEEE Transactions on Circuits and Systems for Video Technology, vol. 99, p. 1, 2020.

[53] M. Yazdi, A. Nedjati, E. Zarei, and R. Abbassi, "A novel extension of DEMATEL approach for probabilistic safety analysis in process systems," Safety Science, vol. 121, pp. 119-136, 2020.

[54] T. Jiang and Y. Liu, "Selective maintenance strategy for systems executing multiple consecutive missions with uncertainty," Reliability Engineering \& System Safety, vol. 193, Article ID 106632, 2020.

[55] K. B. Richard, B. H. Stephen, M. M. Douglas, and S. Eric, "Introduction to unmanned aircraft systems," 2014.

[56] N. A. Golilarz and H. Demirel, "Thresholding neural network (TNN) based noise reduction with a new improved thresholding function," Computational Research Progress in Applied Science \& Engineering, vol. 3, no. 2, pp. 81-84, 2017.

[57] General Aviation Branch of China Air Transport Association, China's Development Report on Civil Drones, General Aviation Branch of China Air Transport Association, Taipei, Taiwan, 2019.

[58] M. Yazdi and S. Kabir, "A fuzzy Bayesian network approach for risk analysis in process industries," Process Safety and Environmental Protection, vol. 111, pp. 507-519, 2017.

[59] N. A. Golilarz, H. Demirel, and H. Gao, "Adaptive generalized Gaussian distribution oriented thresholding function for image de-noising," International Journal of Advanced Computer Science and Applications, vol. 10, no. 2, pp. 10-15, 2019.

[60] Z. Shao, F. Yan, and Z. Zhou, "Path planning for multi-UAV formation rendezvous based on distributed coopera- tive 
particle swarm optimization," Applied Sciences-Base, vol. 16, 2019.

[61] C. Diallo, U. Venkatadri, A. Khatab, and Z. Liu, "Optimal selective maintenance decisions for large serial k-out-of-n: $\mathrm{G}$ systems under imperfect maintenance," Reliability Engineering \& System Safety, vol. 175, pp. 234-245, 2018.

[62] H. Li, C. Guedes Soares, and H.-Z. Huang, "Reliability analysis of a floating offshore wind turbine using Bayesian Networks," Ocean Engineering, vol. 217, Article ID 107827, 2020.

[63] J. Hu, M. Wang, C. Zhao, Q. Pan, and C. Du, "Formation control and collision avoidance for multi-UAV systems based on Voronoi partition," Science China Technological Sciences, vol. 63, no. 1, pp. 65-72, 2020.

[64] J. Hu, H. Zhang, L. Liu, X. Zhu, C. Zhao, and Q. Pan, "Convergent multiagent formation control with collision avoidance," IEEE Transactions on Robotics, vol. 36, no. 6, pp. 1805-1818, 2020.

[65] J.-w. Hu, B.-y. Zheng, C. Wang et al., "A survey on multisensor fusion based obstacle detection for intelligent ground vehicles in off-road environments," Frontiers of Information Technology \& Electronic Engineering, vol. 21, no. 5, pp. 675692, 2020.

[66] J. Zhao, J. Liu, J. Jiang, and F. Gao, "Efficient deployment with geometric analysis for mmWave UAV communications," IEEE Wireless Communications Letters, vol. 9, no. 7, pp. 1115-1119, 2020.

[67] G. Zhu, S. Wang, L. Sun, W. Ge, and X. Zhang, "Output feedback adaptive dynamic surface sliding-mode control for quadrotor UAVs with tracking error constraints," Complexity, vol. 2020, Article ID 8537198, 23 pages, 2020.

[68] J. Wang, P. Zhu, B. He, G. Deng, C. Zhang, and X. Huang, “An adaptive neural sliding mode control with ESO for uncertain nonlinear systems," International Journal of Control, Automation and Systems, vol. 10, p. 1, 2020.

[69] Y. Huang, J. Wang, F. Wang, and B. He, "Event-triggered adaptive finite-time tracking control for full state constraints nonlinear systems with parameter uncertainties and given transient performance," ISA Transactions, vol. 108, pp. 131$143,2020$.

[70] J. Wang, Y. Huang, T. Wang, C. Zhang, and Y. h. Liu, "Fuzzy finite-time stable compensation control for a building structural vibration system with actuator failures," Applied Soft Computing, vol. 93, Article ID 106372, 2020.

[71] Z. Chen, J. Wang, K. Ma, X. Huang, and T. Wang, "Fuzzy adaptive two-bits-triggered control for nonlinear uncertain system with input saturation and output constraint," International Journal of Adaptive Control and Signal Processing, vol. 34, no. 4, pp. 543-559, 2020.

[72] X. Zhang, Y. Wang, X Chen et al., "Decentralized adaptive neural approximated inverse control for a class of large-scale nonlinear hysteretic systems with time delays," IEEE Transactions on Systems, Man, and Cybernetics: Systems, vol. 49, no. 12, pp. 2424-2437, 2018.

[73] J. Liu, C. Wu, G. Wu, and X. Wang, "A novel differential search algorithm and applications for structure design," Applied Mathematics and Computation, vol. 268, pp. 246-269, 2015.

[74] G. Sun, B. Yang, Z. Yang, and G. Xu, "An adaptive differential evolution with combined strategy for global numerical optimization," Soft Computing, vol. 68, pp. 1-20, 2019.

[75] G. Sun, C. Li, and L. Deng, "An adaptive regeneration framework based on search space adjustment for differential evolution," Neural Computing and Applications, pp. 1-7, 2021.
[76] B. Bai, Z. Guo, C. Zhou, W. Zhang, and J. Zhang, "Application of adaptive reliability importance sampling-based extended domain PSO on single mode failure in reliability engineering," Information Sciences, vol. 546, pp. 42-59, 2021.

[77] Y. Cao, Y. Li, G. Zhang, K. Jermsittiparsert, and M. Nasseri, "An efficient terminal voltage control for PEMFC based on an improved version of whale optimization algorithm," Energy Reports, vol. 6, pp. 530-542, 2020.

[78] N. Gao, D. Luo, B. Cheng, and H. Hou, "Teaching-learningbased optimization of a composite metastructure in the $0-10 \mathrm{kHz}$ broadband sound absorption range," The Journal of the Acoustical Society of America, vol. 148, no. 2, pp. EL125-EL129, 2020.

[79] H.-J. Ma, G.-H. Yang, and T. Chen, "Event-Triggered optimal dynamic formation of heterogeneous affine nonlinear multiagent systems," IEEE Transactions on Automatic Control, vol. 66, no. 2, pp. 497-512, 2021.

[80] H. Chen, H. Qiao, L. Xu, Q. Feng, and K. Cai, “A fuzzy optimization strategy for the implementation of RBF LSSVR model in vis-NIR analysis of pomelo maturity," IEEE Transactions on Industrial Informatics, vol. 15, no. 11, pp. 5971-5979, 2019.

[81] S. Qu, Y. Han, Z. Wu, and H. Raza, "Consensus modeling with asymmetric cost based on data-driven robust optimization," Group Decision and Negotiation, pp. 1-38, 2020.

[82] B. Cao, S. Fan, J. Zhao, P. Yang, K. Muhammad, and M. Tanveer, "Quantum-enhanced multiobjective large-scale optimization via parallelism," Swarm and Evolutionary Computation, vol. 57, Article ID 100697, 2020.

[83] B. Cao, J. Zhao, Y. Gu, Y. Ling, and X. Ma, "Applying graphbased differential grouping for multiobjective large-scale optimization," Swarm and Evolutionary Computation, vol. 53, Article ID 100626, 2020.

[84] B. Cao, X. Wang, W. Zhang, H. Song, and Z. Lv, "A manyobjective optimization model of industrial internet of things based on private blockchain," IEEE Network, vol. 34, no. 5, pp. 78-83, 2020.

[85] B. Cao, W. Dong, Z. Lv, Y. Gu, S. Singh, and P. Kumar, "Hybrid microgrid many-objective sizing optimization with fuzzy decision," IEEE Transactions on Fuzzy Systems, vol. 28, no. 11, pp. 2702-2710, 2020.

[86] B. Cao, J. Zhao, P. Yang et al., "Multiobjective 3-D topology optimization of next-generation wireless data center network," IEEE Transactions on Industrial Informatics, vol. 16, no. 5, pp. 3597-3605, 2019.

[87] X. Fu, P. Pace, G. Aloi, L. Yang, and G. Fortino, "Topology optimization against cascading failures on wireless sensor networks using a memetic algorithm," Computer Networks, vol. 177, Article ID 107327, 2020. 